\title{
Use of Fibroquel $\circledast$ (Polymerized type I collagen) in patients with hypoxemic inflammatory pneumonia secondary to COVID- 19 in Veracruz, Mexico
}

\begin{abstract}
Since the beginning of the pandemic, there is currently no specific treatment, however some drugs, after systematic reviews and meta-analysis have been positioned as potential treatments (dexamethasone, tocilizumab, baricitinib), however, other drugs with therapeutic potential are being investigated, such as Polymerized type I collagen (Fibroquel).

Material and methods: Adult patients are admitted to the study from December 2020 to January 2021, with prior informed consent, carriers of viral pneumonia confirmed by axial tomography, secondary to infection by Sars Cov2 virus identified by RT-PCR or antigen test, who also present severity data (dyspnea, desaturation -saturation at room air of $92 \%$ or less-, persistent fever), as well as inflammation markers (D-dimer $1000 \mathrm{ng} / \mathrm{ml} \pm 100$; ferritin> $300 \mathrm{mg}$ ) and that allow treatment with Polymerized type I collagen (Fibroquel ${ }^{\circledR}$ ) at home or in a hospital area.
\end{abstract}

Results: We include data from 35 patients, 19 (54.3\%) women and 16 (45.7\%) men, with a median age of $51.0(38.0-76.0)$ years. The number of patients with a history of previous comorbidities was $34(97.1 \%)$ and $20(57.1 \%)$ had multiple comorbidities (3 or more synchronous comorbidities). The most frequent comorbidities among these patients were obesity in $94.3 \%$, DM2 $62.9 \%$, Arterial hypertension $60.0 \%$, COPD $14.3 \%$, heart disease $8.6 \%$, nephropathy $5.7 \%$, in addition to SLE and trisomy 21 , both in $2.9 \%$ of patients. In the baseline evaluation laboratory, we found that $33(94.3 \%)$ patients had lymphocytopenia and $3(8.6 \%)$ had thrombocytopenia. Median D-Dimer and Ferritin serum levels were $1,200.0(990.0-1,800.0) \mathrm{ng} / \mathrm{mL}$ and $394.5(320.0-492.5) \mathrm{ug} / \mathrm{L}$, respectively. The neutrophillymphocyte index was calculated, which on average was 10.8 (8.0 - 14.7) and an ABCGOALSclx index of $14.0(11.0-16.0)$ was calculated, which translate severe disease and an indication of stay in an intensive care unit. Relation to the pulse oximetry at ambient air in the baseline evaluation, we found that the median $\mathrm{O}_{2}$ saturation was $88.0 \%(86.0 \%$ $-89.0 \%$ ). The median number of days of evolution from the onset of symptoms to the moment in which treatment with Fibroquel was started was 8.0 (7.0-9.0) days. The pulse oximetry quantified 7 days after the start of treatment had a median of $94.0 \%(93.0-95.0 \%)$; Regarding the measurement of prognostic biomarkers, the median d-dimer and ferritin in serum measured at 7 days of follow-up were $656.0(497.0$ - 697.5) ng/mL and 394.5 (320.0492.5) ug/L, respectively.

Conclusion: Polymerized type I collagen (Fibroquel) is an effective drug in patients with inflammatory and hypoxemic pneumonia secondary to Sars COV 2 infection, favoring clinical improvement and favoring extubation, oxygenation, and inflammation indices, as well as a biosafety profile that ensures its use without restrictions in these patients.
Volume I3 Issue I - 202 I

Luis Del Carpio-Orantes,' Sergio GarcíaMéndez, ${ }^{2}$ Jesús Salvador Sánchez-Díaz, ${ }^{3}$

Andrés Aguilar-Silva,' Edna Rosario

Contreras-Sánchez,' Sara Nohemí

Hernández-Hernández'

'Department of Internal Medicine, General Hospital 7I, North

Veracruz Delegation, Instituto Mexicano del Seguro Social, Mexico

${ }^{2} \mathrm{Head}$ of Teaching and Research, Regional Hospital of High Specialty of Oaxaca, Mexico

${ }^{3}$ Critical Care Unit, High Specialty Medical Unit 189, North Veracruz Delegation, Instituto Mexicano del Seguro Social, Mexico

Correspondence: Luis Del Carpio Orantes, Department of Internal Medicine, General Hospital 7I, North Veracruz Delegation, Instituto Mexicano del Seguro Social, Díaz Mirón S/N, Floresta,Veracruz, México, Tel 2292237032,

Email Neurona23@hotmail.com

Received: February 15, 202I | Published: February 242021

Keywords: Type I polymerized collagen, Fibroquel, Pneumonia, COVID-19

\section{Introduction}

After one year of suffering the COVID-19 pandemic with more than $112,000,000$ cases, 2,500,000 deaths and 87,000,000 recovered patients, having identified its etiological agent called Sars Cov2, its pathophysiology as well as molecular, antigenic, and antibody diagnostic strategies, there have been significant difficulties in finding an effective treatment, going through many drugs with an off-label indication (those drugs prescribed under conditions other than those authorized, that is, used for use not reflected in their technical sheet) favored by the aggressive pandemic environment and the multiple deaths reported, however today we continue without specific treatment. ${ }^{1,2}$

The few drugs that have shown utility, after a large analysis of the information and after randomized controlled studies, as well as systematic reviews and meta-analyzes, are: dexamethasone and enoxaparin (favored by the recovery study that excluded azithromycin, hydroxychloroquine, lopinavir/ritonavir, and convalescent plasma); Antivirals are highly questionable, with the apparent efficacy of remdesivir, and it has been shown that others such as oseltamivir, lopinavir/ritonavir, and antibiotics, can add morbidity and mortality in non-severe cases, which is why they are not currently routinely recommended. ${ }^{3-6}$ Other drugs that initially seemed to be effective have been displaced even by their own pharmaceutical companies because there was no full indication to support the improvement in terms of patient mortality, although other variables such as bed days, avoiding intubation, or the requirement of amines in critically ill patients, etc., such as the case of tocilizumab (although recently Recovery itself has returned it to the therapeutic arsenal) and ivermectin. ${ }^{7-9}$

Recently, some experiences have been published with drugs with off-label indication, such as ruxolitinib and baricitinib, which are inhibitors of the Janus pathways (JAK1 and JAK2) that decrease pro- 
inflammatory cytokines, however with risks of immunosuppression that could condition added infections that they add morbidity and mortality to patients, mainly in combination with other immunosuppressants such as steroids. ${ }^{10-12}$ Another drug that appears to have demonstrated utility in a Canadian study is colchicine, which demonstrated utility in preventing progression to severe forms of the disease (Colcorona study). ${ }^{13}$

Recently, one of the most prestigious institutes in Mexico, Instituto Nacional de Ciencias Médicas y Nutrición "Salvador Zubirán", has established a protocol using a drug called polyvinyl pyrrolidone collagen or Polymerized type I collagen (Fibroquel ${ }^{\circledR}$, Aspid),${ }^{14}$ which, due to its properties seen in rheumatological pathologies (rheumatoid arthritis, osteoarthritis) against immunity-mediated inflammation, has begun its usefulness in this entity. The proposed dose is $3 \mathrm{ml}$ daily intramuscular, from day 1 to 3 and $1.5 \mathrm{ml}$ daily from day 4 to 7 , being a 7-day treatment cycle, which in severe and/or persistent cases can be extended to two cycles; It has been used in cases of inflammatory pneumonia secondary to COVID-19, taking advantage of its various properties, which are:

i. Negatively modulate the expression of IL-1 $\beta$, IL-8, TNF-a, TGF- $\beta 1$, IL-17, Cox-1, and leukocyte adhesion molecules (ELAM-1, VCAM-1 and ICAM-1), ${ }^{15,16}$

ii. Significantly increases the mediators and modulating mechanisms of inflammation (expression of IL-10 and the number of regulatory T cells). ${ }^{17}$

iii. Reduces tissue fibrosis, without producing adverse effects. ${ }^{17}$

Due to these properties, it has been theorized that, due to its systemic effect, it could have some impact on the negative regulation of the expression of the pro-inflammatory cytokine storm, in the number of effector T cells Th1, Th17, Th22, adhesion molecules leukocyte and increase IL-10 and the number of regulatory T cells, which can lead to important benefits for the treatment of the hyperinflammatory phase and probably in the acute respiratory distress syndrome that patients with mild to severe COVID-19 present with a biosecurity profile devoid of severe adverse effects. ${ }^{18,19}$

\section{Material and methods}

Adult patients are admitted to the study from December 2020 to January 2021, with prior informed consent, carriers of viral pneumonia confirmed by axial tomography, secondary to infection by Sars Cov2 virus identified by RT-PCR or antigen test, who also present severity data (dyspnea, desaturation -saturation at room air of $92 \%$ or less-, persistent fever), as well as inflammation markers (D-dimer 1000ng/ $\mathrm{ml} \pm 100$; ferritin $>300 \mathrm{mg}$ ) and that allow treatment with Polymerized type I collagen (Fibroquel ${ }^{\circledR}$ ) at home or in a hospital area.

Age, gender, comorbidities, characteristics of hematic cytometry, oxygen saturation, D-dimer and ferritin were evaluated at baseline and at 7 days of treatment; home vs. hospital stay; oxygen therapy by non-invasive ventilation (oxygen through nasal tips or reservoir mask) vs invasive (mechanical ventilation), as well as the use of one or more cycles of the drug and finally its evolution (clinical, biochemical and oxygenation improvement) and prognosis. As part of the severity, the neutrophil-lymphocyte index is calculated as well as the score ABC-GOALSclx. Non-adult patients, pregnant women and those who do not consent to the application of the drug are excluded. The recommended intradermal reaction is only practiced prior to the use of the drug in those patients who have a history of prior drug allergies or anaphylaxis. A record of possible adverse events will be kept. At the time of entering the study, previous treatments are suspended, even those that have proven efficacy in previous studies, such as the case of steroids (dexamethasone, prednisone), which in this case, is considered a therapeutic failure. Only common analgesics and antipyretics, anticoagulants (LMWH or direct oral anticoagulants) are allowed during the cycle with Fibroquel ${ }^{\circledR}$

\section{Results}

We include data from 35 patients, $19(54.3 \%)$ women and 16 $(45.7 \%)$ men, with a median age of $51.0(38.0$ - 76.0) years. The number of patients with a history of previous comorbidities was $34(97.1 \%)$ and $20(57.1 \%)$ had multiple comorbidities (3 or more synchronous comorbidities). The most frequent comorbidities among these patients were obesity in $94.3 \%$, DM2 62.9\%, Arterial hypertension $60.0 \%$, COPD $14.3 \%$, heart disease $8.6 \%$, nephropathy $5.7 \%$, in addition to SLE and trisomy 21 , both $2.9 \%$ of patients.

Regarding the use of medications prior to the baseline evaluation, we found that all patients had received some medication for the treatment of their condition. The medications that were indicated most frequently to this group of patients were the following, in order of frequency we found azithromycin in the first place with $77.1 \%$, followed by ivermectin with $51.4 \%$, dexamethasone in $45.7 \%$, prednisone in $45.7 \%$, ceftriaxone in $31.4 \%$ and oseltamivir in $28.6 \%$ of the patients; The other medications that the patients received and their frequencies are found in Table 1. The median number of medications received prior to the initiation of polyvinylpyrrolidone collagen was 3.0(3.0-4.0). In the baseline evaluation laboratory, we found that $33(94.3 \%)$ patients had lymphocytopenia and 3(8.6\%) had thrombocytopenia. Median D-Dimer and Ferritin serum levels were 1,200.0 (990.0 - 1,800.0) ng/mL and 394.5 (320.0-492.5) ug/L, respectively. The neutrophil-lymphocyte index was calculated, which on average was 10.8(8.0-14.7) and an ABC-GOALSclx index of 14.0(11.0-16.0) was calculated, which translate severe disease and an indication of stay in an intensive care unit.

In relation to the pulse oximetry at ambient air in the baseline evaluation, we found that the median $\mathrm{O}_{2}$ saturation was $88.0 \%$ $(86.0 \%-89.0 \%)$.

The median number of days of evolution from the onset of symptoms to the moment in which treatment with Fibroquel was started was 8.0(7.0-9.0) days.

\section{Measurement of clinical and laboratory variables during the 7-day follow-up}

Regarding the treatment with Fibroquel, 34(97.1\%) patients received one treatment cycle with this drug and $1(2.9 \%)$ patients received two treatment cycles. Regarding the site where they were treated, 31(88.6\%) patients received home treatment and $4(11.4 \%)$ patients had hospital treatment. No patient reported a history of severe allergies or anaphylaxis, so the intradermal reaction was not carried out. No infectious or anaphylactic adverse events were reported associated with the use of Fibroquel, the main complaint reported was pain at the application site in $40 \%$ that improved with the addition of simple lidocaine to intramuscular injection.

The pulse oximetry quantified 7 days after the start of treatment had a median of $94.0 \%(93.0-95.0 \%)$; regarding the measurement of prognostic biomarkers, the median d-dimer and ferritin in serum measured at 7 days of follow-up were $656.0(497.0-697.5) \mathrm{ng} / \mathrm{mL}$ and 394.5(320.0-492.5) ug/L, respectively. Regarding the need for supplemental oxygen, $94 \%$ of the patients used oxygen at the beginning of Fibroquel therapy, with variable requirements from 3 to 10 liters continuously $\left(\mathrm{FiO}_{2}\right.$ variable $\left.30-60 \%\right)$, at the end of it. Only 
$20 \%$ continued to use oxygen, managing to do without it between days $7-14$. Only 2 patients required intubation, managing to be extubated after the end of the fibroquel cycle. Regarding the outcomes measured in this analysis, we find that $34(97.1 \%)$ patients had improvement in their clinical conditions and $1(2.9 \%)$ patient died from complications of this disease.

Table I Results of clinical and laboratory variables of patients with severe COVIDI9 who were treated with polymerized type I collagen (Fibroquel)

\begin{tabular}{|c|c|}
\hline Variable* & Results N = 35 \\
\hline \multicolumn{2}{|l|}{ Demographic variables and previous comorbidities } \\
\hline \multicolumn{2}{|l|}{ Gender } \\
\hline Women & $19(54.3)$ \\
\hline Men & $16(45.7)$ \\
\hline Age, years; median (IQR) & $51.0(38.0-76.0)$ \\
\hline \multicolumn{2}{|l|}{ Previous comorbidities } \\
\hline Obesity & $33(94.3)$ \\
\hline Diabetes mellitus type 2 & $22(62.9)$ \\
\hline Systemic hypertension & $21(60.0)$ \\
\hline Chronic obstructive pulmonary disease & $5(14.3)$ \\
\hline Heart disease & $3(8.6)$ \\
\hline Chronic kidney disease & $2(5.7)$ \\
\hline Systemic lupus erythematosus & I (2.9) \\
\hline Trisomy 21 & I (2.9) \\
\hline \multicolumn{2}{|l|}{ Medications used prior to baseline evaluation } \\
\hline Azithromycin & 27 (77.1) \\
\hline Ivermectin & $18(5 \mid .4)$ \\
\hline Prednisone & $16(45.7)$ \\
\hline Dexamethasone & $16(45.7)$ \\
\hline Ceftriaxone & II (3I.4) \\
\hline Oseltamivir & $10(28.6)$ \\
\hline Enoxaparin & $5(14.3)$ \\
\hline Baricitinib & I (2.9) \\
\hline Paracetamol & I (2.9) \\
\hline Ribavirin & I (2.9) \\
\hline Acetylsalicylic acid & $\mathrm{I}(2.9)$ \\
\hline Rivaroxaban & I (2.9) \\
\hline Levofloxacin & I (2.9) \\
\hline Moxifloxacin & I (2.9) \\
\hline \multicolumn{2}{|l|}{ Baseline evaluation laboratory } \\
\hline Lymphocytopenia & $33(94.3)$ \\
\hline Thrombocytopenia & $3(8.6)$ \\
\hline Neutrophil-lymphocyte index (IQR) & $10.8(8.0-14.7)$ \\
\hline D-dimer, ng / mL; median (IQR) & $1,200.0(990.0-1,800.0)$ \\
\hline Serum ferritin, ng / mL; median (IQR) & $790.0(670.5-1,000.0)$ \\
\hline ABC-GOALS clx (RIQ) & $14.0(11.0-16.0)$ \\
\hline \multicolumn{2}{|l|}{ Pulse oximetry baseline evaluation } \\
\hline Medium ambient air pulse oximetry (IQR); \% O2 Sat & $88.0(86.0-89.0)$ \\
\hline Patients using oxygen at the beginning of fibroquel\% & 94.00 \\
\hline \multicolumn{2}{|l|}{ Variables associated with treatment with Fibroquel } \\
\hline Day of onset, median (IQR) & $8.0(7.0-9.0)$ \\
\hline Collagen cycles, median (IQR) & $1.0(1.0-1.0)$ \\
\hline \multicolumn{2}{|l|}{ Type of oxygen therapy } \\
\hline Non invasive & $33(94.3)$ \\
\hline Invasive & $2(5.7)$ \\
\hline \multicolumn{2}{|l|}{ Laboratory at 7 days of follow-up } \\
\hline D-dimer, ng / mL; median (IQR) & $656.0(497.0-697.5)$ \\
\hline Serum ferritin, ng / mL; median (IQR) & $394.5(320.0-492.5)$ \\
\hline \multicolumn{2}{|l|}{ Pulse oximetry at 7 days of follow-up } \\
\hline Medium ambient air pulse oximetry (IQR); \% O2 Sat & $94.0(93.0-95.0)$ \\
\hline Patients using oxygen after fibroquel\% & 20.00 \\
\hline \multicolumn{2}{|l|}{ Treatment site } \\
\hline Home & $31(88.6)$ \\
\hline Hospital & $4(11.4)$ \\
\hline \multicolumn{2}{|l|}{ Outcome } \\
\hline Improvement & 34 (97.I) \\
\hline Death & I (2.9) \\
\hline
\end{tabular}

* Results expressed with $\mathrm{n}(\%)$, except when something different is specified. 


\section{Comparative analysis of pulse oximetry, serum levels of d-dimer and ferritin, quantified in the baseline evaluation VS the measurement at $\mathbf{7}$ days of follow-up}

In this analysis, we found that the pulse oximetry measured at 7 days of evolution was significantly higher compared to the pulse oximetry measured in the baseline evaluation $[88.0(86.0$ - 89.0)\% VS $94.0(93.0-95.0) \% ; \mathrm{p}=0.001)]$ (Figure 1A). On the other hand, the median d-dimer and ferritin in serum measured at 7 days of follow-up, were significantly lower compared to the medians of the measurements of these biomarkers that were performed in the baseline evaluation [1,200 (990.0 - 1,800.0) ng/mL VS $656.0(497.0$ - 697.5) mg/mL; p $=0.001]$ and $[790.0(670.5-1,000) \mathrm{ug} / \mathrm{mL}$ VS $394.5(320.0-492.5)$; $\mathrm{p}=0.001$ ), respectively (Figures $1 \mathrm{~B}$ and $1 \mathrm{C}$ ).

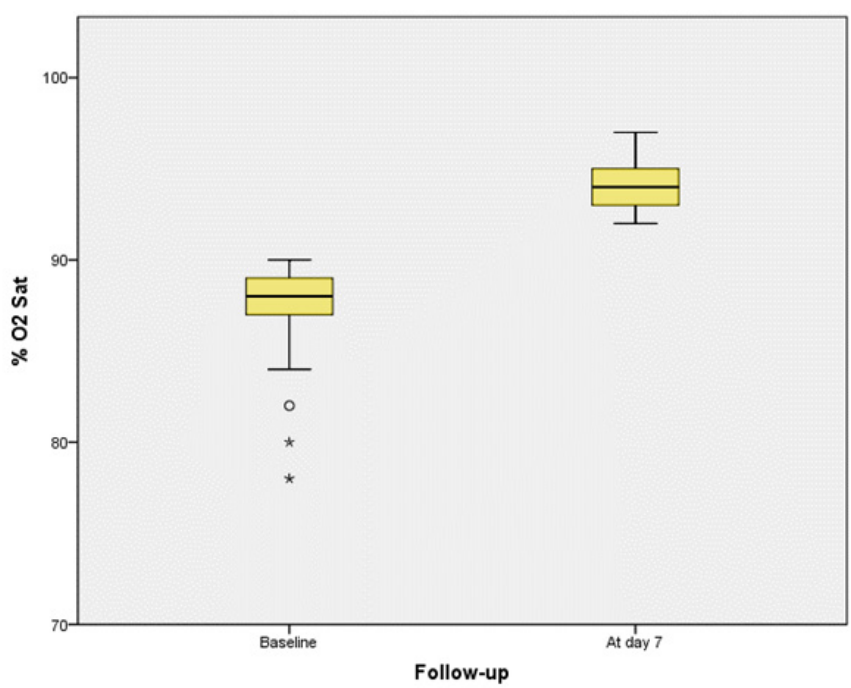

Figure IA Box and whisker plot with the differences in\% O2 Sat measured in the baseline evaluation and in the 7-day follow-up *.

$*(p=0.001$, estimated with Wilcoxon rank test $)$.

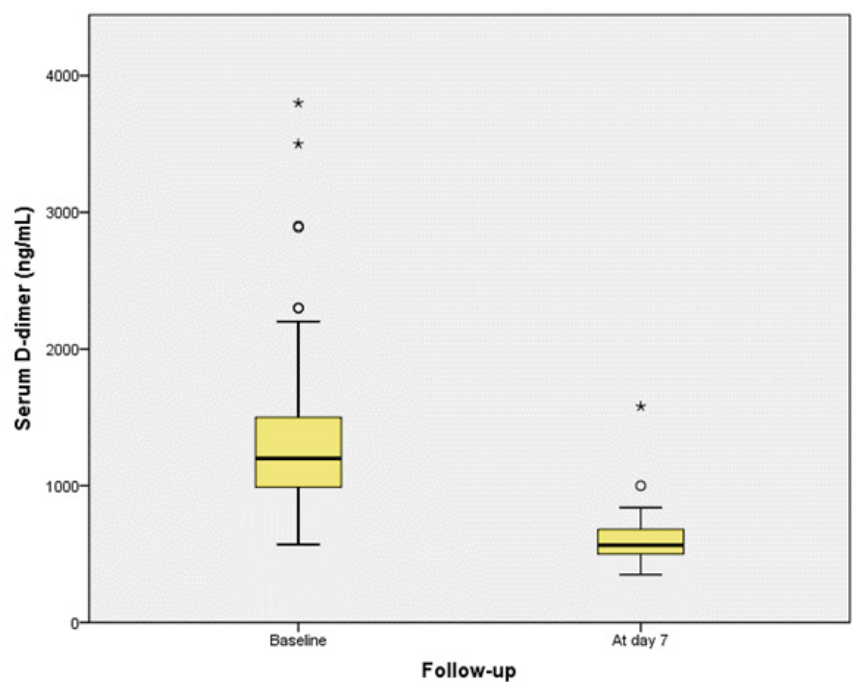

Figure IB Box-and-whisker plot with the differences in serum levels of d-dimer measured in the baseline evaluation and in the 7-day follow-up *

$*(p=0.00 \mathrm{I}$, estimated with Wilcoxon rank test $)$.

\section{Discussion}

In these patients, the treatment with Fibroquel is shown to be encouraging for the treatment of patients with various risk factors that present with severe inflammatory and hypoxemic pneumonia, with criteria of stay in an intensive care unit, oxygen users and that probably require invasive mechanical ventilation. However, after the use of Fibroquel, the inflammation and oxygenation markers improved considerably, avoiding hospitalizations as well as the oxygen requirements, as well as the management of critically ill patients favoring extubation (ventilatory, amines, etc.), something comparable to the use of other treatments such as tocilizumab or baricitinib, however in these patients who received Fibroquel without secondary adverse effects (mainly allergies or secondary infections), the main discomfort being a pain at the application site, which made it have a higher biosecurity profile.

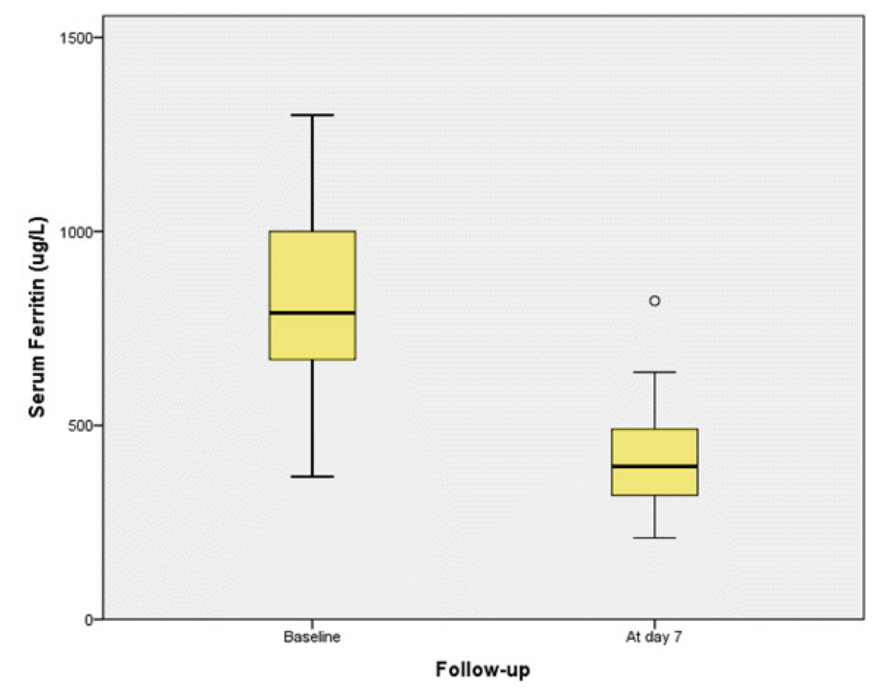

Figure IC Box-and-whisker plot with differences in serum ferritin levels measured in the baseline evaluation and in the 7-day follow-up *.

$*(p=0.001$, estimated with Wilcoxon rank test $)$.

It is important to note that these patients presented poor evolution despite the use of steroids, which are current medications with a precise indication in this type of pneumonia (supported by the Recovery study) and that on the day of the evaluation (day 8 on average), considered therapeutic failure of the same, so its use is discontinued, which helps to avoid greater immunosuppression that can subsequently lead to added infections and allows evaluating the effect of Fibroquel without other immunomodulators.

\section{Conclusion}

Polymerized type I collagen (Fibroquel) is an effective drug in patients with inflammatory and hypoxemic pneumonia secondary to Sars Cov2 infection, favoring clinical improvement and favoring extubation, oxygenation and inflammation indices, as well as a biosafety profile that ensures its use without restrictions in these patients.

\section{Conflicts of interest}

The authors declare there are no conflicts of interest.

\section{Acknowledgments}

The authors thank Dr. Janette Guadalupe Furuzawa Carballeda and Dr. Hilda Adriana Castro Rocha from the Instituto Nacional de Ciencias Médicas y Nutrición "Salvador Zubirán" for their support at all times to bring this research to fruition. 


\section{References}

1. Del Carpio-Orantes L, González-Segovia O, Mojica-Ríos F, et al. Neumonía grave por COVID-19 curada con prono consciente y tocilizumab. Comunicación de un caso y revisión de la evidencia terapéutica farmacológica. Med Int Méx. 2020;36(4):585-595.

2. González Vázquez C, López Guzmán J. Medicamentos off-label: cuestiones terminológicas y conceptuales. Index de Enfermería. 2017;26(4):292-294.

3. Horby P, Lim WS, Emberson JR, et al. Dexamethasone in Hospitalized Patients with Covid-19 - Preliminary Report. N Engl J Med. 2020.

4. Beigel JH, Tomashek KM, Dodd LE, et al. Remdesivir for the Treatment of Covid-19 - Final Report. N Engl J Med. 2020;383(19):1813-1826.

5. Billett HH, Reyes-Gil M, Szymanski J, et al. Anticoagulation in COVID-19: Effect of Enoxaparin, Heparin, and Apixaban on Mortality. Thromb Haemost. 2020;120(12):1691-1699.

6. Javier Mancilla-Galindo, Jorge Óscar García-Méndez, Jessica MárquezSánchez, et al. All-cause mortality among patients treated with repurposed antivirals and antibiotics for COVID-19 in Mexico City: A Real-World Observational Study. Med Rxiv. 2020.

7. Furlow B. COVACTA trial raises questions about tocilizumab's benefit in COVID-19. The Lancet Rheumatology. 2020;2(10):e592.

8. https://www.merck.com/news/merck-statement-on-ivermectin-useduring-the-covid-19-pandemic/

9. https://www.idsociety.org/practice-guideline/covid-19-guidelinetreatment-and-management/\#toc-14

10. Ovilla-Martínez R, Cota-Rangel X, De La Peña-Celaya JA, et al. Ruxolitinib como tratamiento contra el COVID-19 en población mexicana. Rev Hematol Mex. 2020;21(4):195-204.

11. Kalil AC, Patterson TF, Mehta AK, et al. Baricitinib plus Remdesivir for Hospitalized Adults with Covid-19. N Engl J Med. 2020.
12. Richardson $P$, Griffin I, Tucker C, et al. Baricitinib as potential treatment for 2019-nCoV acute respiratory disease. Lancet. 2020;395(10223):e30 e31.

13. Jean-Claude T, Nadia Bouabdallaoui, Philippe L L'Allier, et al. Efficacy of Colchicine in Non-Hospitalized Patients with COVID-19. medRxiv. 2021 .

14. https://ichgcp.net/es/clinical-trials-registry/NCT04517162

15. Furuzawa-Carballeda J, Fenutria-Ausmequet R, Gil-Espinosa V, et al. Polymerized-type I collagen for the treatment of patients with rheumatoid arthritis. Effect of intramuscular administration in a double blind placebo-controlled clinical trial. Clin Exp Rheumatol. 2006;24(5):514-520.

16. Furuzawa-Carballeda J, Krötzsch E, Barile-Fabris L, et al. Subcutaneous administration of collagen-polyvinylpyrrolidone down regulates IL1beta, TNF-alpha, TGF-beta1, ELAM-1 and VCAM-1 expression in scleroderma skin lesions. Clin Exp Dermatol. 2005;30(1):83-86.

17. Furuzawa-Carballeda J, Rodríguez-Calderón R, Díaz de León L, et al. Mediators of inflammation are down-regulated while apoptosis is up-regulated in rheumatoid arthritis synovial tissue by polymerized collagen. Clin Exp Immunol. 2002;130(1):140-149.

18. Furuzawa-Carballeda J, Ortíz-Ávalos M, Lima G, et al. Subcutaneous administration of polymerized type I collagen downregulates interleukin (IL)-17A, IL-22 and transforming growth factor- $\beta 1$ expression, and increases Foxp3-expressing cells in localized scleroderma. Clin Exp Dermatol. 2012;37(6):599-609.

19. Furuzawa-Carballeda J, Rojas E, Valverde M, et al. Cellular and humoral responses to collagen-polyvinylpyrrolidone administered during short and long periods in humans. Can J Physiol Pharmacol. 2003;81(11):1029-1035. 